\title{
VECTOR QUANTISATION BASED IMAGE ENHANCEMENT
}

\author{
W. Paul Cockshott, Sumitha L. Balasuriya, Irwan Prasetya Gunawan, J. Paul Siebert \\ University of Glasgow, Computing Science Department \\ 17 Lilybank Gardens, Glasgow G12 8QQ \\ \{wpc,sumitha,ipguna,psiebert\}@dcs.gla.ac.uk
}

Keywords: Image rescaling, bspline, vector quantization, fractal

\begin{abstract}
We present a new algorithm for rescaling images inspired by fractal coding. It uses a statistical model of the relationship between detail at different scales of the image to interpolate detail at one octave above the highest spatial frequency in the original image. We compare it with Bspline and bilinear interpolation techniques and show that it yields a sharper looking rescaled image.
\end{abstract}

\section{INTRODUCTION}

Digital cinema sequences can be captured at a number of different resolutions, for example $2 \mathrm{~K}$ pixels accross or $4 \mathrm{~K}$ pixels accross. The cameras used for high resolutions are expensive and the data files they produce are large. Because of this, studios may chose to capture some sequences at lower resolution and others at high resolution. The different resolution sequences are later merged during post production. The merger requires that some form of image expansion be performed on the lower resolution sequences. In this paper we present a new method for doing the image expansion that has some advantages over the orthodox bilinear or bicubic interpolation methods.

\subsection{Traditional approach}

If you scale up a $2 \mathrm{~K}$ pixel image $A$ to a new image $B$ which is $4 \mathrm{~K}$ pixels accross, each original pixel is being replaced with 4 pixels. But you have no additional information as to what these 4 pixels should contain. If you scale up by interpolation, then the new pixels in $B$ are generated by some polynomial function over the corresponding the neighbourhood in the smaller input image $A$. A consequence of this is that the scaled up image looks smoother and contains less energy at the highest spatial frequencies than the original from which it was derived. When one scales up, one creates new higher spatial frequency bands with no information as to what they should contain.

\subsection{Fractal Enhancement}

An alternative approach, fractal encoding, originally reported by Barnsley(Barnsley and Hurd, 1993), allows rescaled images to contain new high frequency information. Fractal encoding takes advantage of the self similarity across scales of natural scenes. A fractal code for an image consists of a set of contractive affine maps from the image, onto the image. Taken as a whole, these maps compose a collage such that each pixel is mapped onto by at least one such map. The maps operate both in the spatial and the luminance domain. In the luminance domain they specify a target pixel $p$ by an equation of the form $p=a+b q$ where $q$ is the mean brightness of a downsample region of source pixels. In the spatial domain they specify the coordinates of the source pixels supporting $q$ as the result of rotation, scaling and translation operations on the coordinates of the destination pixels.

The image is regenerated from the codes by iterated application of the affine maps. The iteration process has an attractor that is the output image. If the maps have been well chosen this attractor approximates well to a chosen input image.

A particular fractal code might specify each $4 \times 4$ rectangle within a $256 \times 256$ pixel output image in terms of a contractive map on some $8 \times 8$ rectangle at some other point in the image. As the iteration pro- 


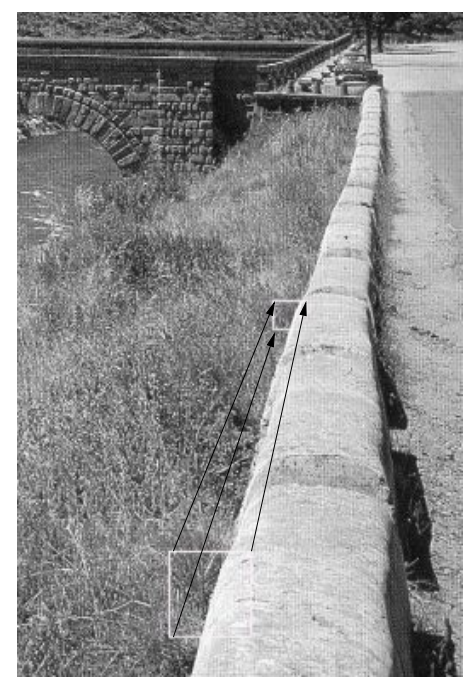

Figure 1: Illustration of how shrinking is used to fill in detail in fractal enhancement.

ceeds higher and higher frequency information is built up. If we start from a uniform grey image, the first iteration will generate detail at a spatial frequency of 8 pixels. After one iteration source blocks of 8 pixels accross will contain up to one spatial wave. After the second interpolation these waves will have been shifted up in frequency to 4 pixels accross. Each iteration adds detail one octave higher until the Nyquist limit of the output image is reached: 128 spatial cycles in this case.

It is evident that if we specify the contractive mappings relative to the scale of the whole image rather than in terms of pixels, then the same set of mappings could be used to generate a $512 \times 512$ pixel output image. In this case the contractive mappings would shrink 16 pixel blocks to 8 pixel blocks. After an an additional round of iteration the 512 pixel output image will contain spatial frequencies up to 256 cycles.

Fractal codes can thus be used to expand an image, generating new and higher spatial frequencies in the process. Although the additional detail that is added by this process can not have been available in the source image it nevertheless 'looks plausible' because the 'new' details are scaled down versions of details that were present in the original picture (see Figure 1). The search process used in a fractal encoder scans a half sized copy of the original image to find a match for each small block in the original image. In fractal enhancement the small blocks are then replaced by their full sized corresponding blocks. The detail enhancement comes because there is a systematic relationship between the low frequency and high frequency information within blocks. This al- lows high frequency information in a larger block to be plausibly substituted into a smaller block when that is enlarged.

Fractal codes however suffer from two serious obstacles to their widespread adoption: the encoding algorithm is slow and their general use is blocked by patent restrictions. In this paper we present an alternative approach that learns lessons from fractal coding but avoids these difficulties. Instead of using fractals we use vector quantisation to enhance the detail of an image.

\section{OUR ALGORITHM}

The key idea of our approach is that because there is a systematic relationship between low and high frequency information within a neighbourhood, it should be possible for a machine learning algorithm to discover what this relationship is and exploit this knowledge when enhancing an image. We use vector quantisation to categorise areas of the image at different scales, learn the systematic relationship between the coding of corresponding areas at varying scales, and then use this information to extrapolate a more detailed image. The entire process works by

1. Running a training algorithm to learn the crossscale structure relations in example pictures. In the experiments here two images were used one from the 'face' sequence and one from the 'trees' sequence.

2. Using this information to automatically construct a new image enhancing program.

3. Applying the enhancing program to digital cine images to generate new images at twice the resolution.

\subsection{The Training Algorithm}

The aim of the training algorithm is to learn what high frequency detail is likely to be associated with the low frequency features at a given point in an image. Given an image $I$ we construct a half sized version of the image $I_{0.5}$ and expand this to form a new blurred image $I_{b}$ which is the original size, by using linear interpolation. We now form a difference image $I_{d}=I-I_{b}$ which contains only the high frequency details.

It it clear that we have a genetive association between position $I_{0.5}[x, y]$ and the four pixel block $G_{x, y}=\left\{I_{d}[2 x, 2 y], I_{d}[2 x+1,2 y], I_{d}[2 x, 2 y+\right.$ $\left.1], I_{d}[2 x+1,2 y+1]\right\}$. We aim to categorise the regions around each position in $I_{0.5}[x, y]$, categorise 
the corresponding blocks $G_{x, y}$ and learn the associations between these categories.

\subsubsection{Categorising the Upper Layer}

Associate with each pixel $p \in I_{0.5}$ a neighbourhood and compute the differences between $p$ and its neighbours. These define a 4 element vector. Using the algorithm given in (Linde et al., 1980) construct a vector quantisation codebook $B_{1}$ for these features. Assume that the code book has $n$ entries.

\subsubsection{Categorising the Lower Layer}

Use the same vector quantisation algorithm to construct a second vector quantisation codebook $B_{2}$ for the set of vectors $G_{x, y}$. Assume that the code book again has $n$ entries.

\subsubsection{Learning the Association}

Encode the neighbourhoods around each pixel $p \in$ $I_{0.5}$ with $B_{1}$ to yield an encoded image $E_{0.5}$. Encode each $G_{x, y}$ associated with each pixel $p \in I_{0.5}$ with $B_{2}$ to yield an encoded image $E_{b}$. The entries in both the encoded images are indexes into the respective codebooks.

Construct a $n \times n$ frequency table $F$ that counts how frequently each code from $B_{1}$ is associated with each code from $B_{2}$. Finally convert the frequency table to a conditional probability table by dividing by the number of observations.

\subsection{The Program Generator}

The aim of the program generator is to take the tables $B_{1}, B_{2}, F$ and use them to generate pascal libraries that can be used to index and predict detail in subsequent images. The process is analogous to the way Lex(Levine et al., 1992) constructs scanner tables in $\mathrm{C}$ from a regular grammar.

Two optimisations are performed prior to outputing the tables:

1. Table $F$ is converted from a conditional probability table to a table encoding the cumulative probability of each entry in $B_{2}$ being associated with and entry in $B_{1}$.

2. Hierarchical Vector Quantisation (Chaddha et al., 1995) indices are constructed for the two codebooks to enable future encoding to be of $\mathbf{O}(4)$ rather than $\mathbf{O}(\mathrm{n})$.

\subsection{The Enhancement Algorithm}

The enhancement program has the library produced above linked to it. The aim of the program is to read in an image $J$ and produce an image $J_{2}$ of twice the size with enhanced detail.

Create image $J_{b}$ of twice the size of $J$ using linear interpolation.

Create an empty image $J_{d}$ twice the size of $J$.

For each pixel $p \in J$ at position $x, y$ compute its differences with its four neighbours as described in 2.1.1. Encode the four differences using a vector quantisation index for book $B_{1}$ to yield a code index number $i$. Select the $i$ th row of $F$. Draw a real number $r$ at random such that $0 \leq r<1$. Scan row $F[i]$ until an $F[i, j]>r$ is found. Select the 4 element vector $B_{2}[j]$. Place this vector in the image $J_{d}$ at positions $\left\{J_{d}[2 x, 2 y], J_{d}[2 x+1,2 y], J_{d}[2 x, 2 y+1], J_{d}[2 x+\right.$ $1,2 y+1]\}$.

Once this process has been completed for each pixel in $J$ the image $J_{d}$ contains details whose spatial frequency is one octave higher than those that are represented in $J_{b}$. Each detail occurs with the same probability with respect to the categorisation of localities in $J$ as details occured in $I_{d}$ with respect to the categorisation of localities in $I_{0.5}$.

The final step forms the enhanced image $J_{e}$ by the operation $J_{e}=J_{b}+J_{d}$

\section{RESULTS}

Our experiments were conducted on $1920 \times 1080$ video frames in the DPX image format captured by a Thompson Viper D-Cinema video camera. The pixels were in 10 bit logarithmic format. Image expansion using our system was compared to conventional bilinear interpolation and B-Spline interpolation techniques. The experimental procedure can be described as follows:

1. The enhancement system was trained on a $1920 \times$ 1080 DPX frame from an outdoor sequence. The test images used were a studio frame (Figure 2) and a later frame from the outdoor sequence from which original training frame had been selected (Figure 5). The training and test frame from this sequence had different zoom settings, the training frame having had a higher zoom factor than the second test frame.

2. The images were downsampled using bilinear interpolation to $960 \times 540$ and output in DPX format. 
3. Image expansion to double the original resolution of $1920 \times 1080$ was performed using our algorithm, bilinear and bspline interpolations.

4. Original and expanded DPX video frames were compared subjectively based on perceived detail in image patches. The quality of reproduction was also evaluated objectively using several image quality metrics described below.

First, a traditional measure based on Peak Signalto-Noise Ratio (PSNR) (Pratt, 1978) was calculated. In this paper, the PSNR was calculated on the 10bit logarithmic representation of pixel values. This metric is very practical and easy to compute, however it does not always correlate well with the quality perceived by human users (Girod, 1993). An alternative using a modified version of the PSNR based on perceived visibility of error, namely Weighted Peak Signal-to-Noise Ratio (WPSNR) (Voloshynovskiy et al., 1999), was also computed. In this metric, error on textured area would be given less weighting factor than that on flat surface.

Since image expansion algorithms usually introduce blur artifacts, another quality metric (Gunawan and Ghanbari, 2005) which is able to detect and measure the degree of blurriness on image was also used. This metric uses features extracted from the frequency domain through two-dimensional Discrete Fourier Transfrom (DFT) computation over a localised area on the gradient image. In an image contaminated by blurring distortion, some frequency components appear attenuated when compared to the same set of components on the original image. Blurriness detection can be done by analysing the decay in the strength of these frequency components. One quality parameter produced by this metric called harmonic loss, is a relative comparison of certain frequency components from different images. This parameter can be used to measure blurring on image.

It is subjectively apparent that our algorithm has regenerated plausible image detail that was irretrievable when using the B-Spline and Bilinear interpolation approaches (Figure 3). The down-sampling suppressed visual information which only our algorithm could recover based on its knowledge of statistical cooccurrence of low and high frequency image content.

Objective comparison of our algorithm with $\mathrm{Bi}$ linear and B-Spline interpolation (Table 1) for image expansion shows a marked improvement in the PSNR and WPSNR metrics for our algorithm. Bilinear interpolation performs marginally better than B-Spline interpolation and our algorithm has almost twice the objective image quality score as the second best approach.

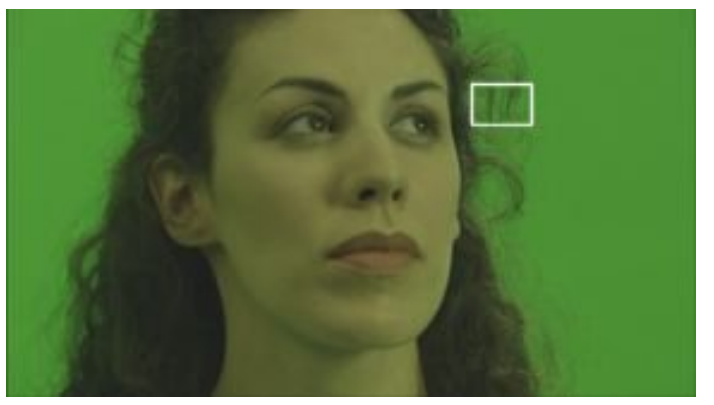

Figure 2: Reduced scale colour image from original DPX digital cine frame from studio sequence 'face'. Relatively soft focus is used with a moving subject. Box indicates where detail is shown in Figure 3. Note that this and all following images are uncorrected log colour space.

It was observed that VQ-based enhancement method was better than conventional method (e.g bspline) since the latter introduces more blurriness to the images. As an illustration, Figure 4 compares the degree of the blurriness (expressed as blur index) of several images from 'outdoor' sequence which have been enhanced by three different methods (our proposed VQ-based, bilinear, and b-spline). Note that higher value of the blur index on an image implies that the image contains more blurring artifacts. It shows that the blurriness indices of the bspline and bilinear enhanced images are generally higher than those of VQ-based. Figures 6 and 7 show the relative blurriness of the expanded images compared to the original.

\section{CONCLUSION}

This paper presented a novel approach to image enhancement using a technique which would avoid the known shortcomings of fractal enhancement. We learnt the statistical properties of the co-occurrence of low and high frequency image content and used these probability distributions to predict image content during image expansion. Our algorithm out per-

Table 1: Peak-signal-to-noise ratio and weighted-peaksignal-to-noise ratio image quality metrics for the image expansions shewn in Figure 3. (1) Our algorithm, (2) Bilinear Interpolation, (3)B-Spline interpolation. Note that all calculations are done on the 10 bit logarithmic representation used in DPX which compresses the upper part of the dynamic range and tends to give lower PSNR values than will be familiar from for 8 bit linear representations.

\begin{tabular}{|c|c|c|c|}
\hline & $(1)$ & $(2)$. & $(3)$ \\
\hline \hline PSNR & $16.8 \mathrm{~dB}$ & $8.4 \mathrm{~dB}$ & $6.4 \mathrm{~dB}$ \\
\hline WPSNR & $22.9 \mathrm{~dB}$ & $14.4 \mathrm{~dB}$ & $12.4 \mathrm{~dB}$ \\
\hline
\end{tabular}




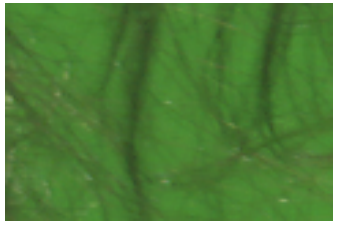

(a) Original

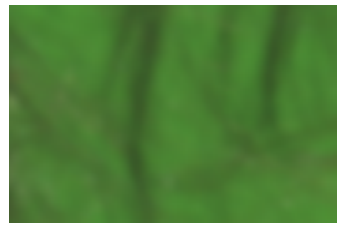

(c) Bspline

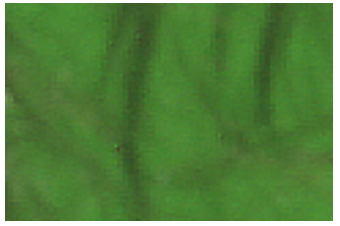

(b) Our algorithm

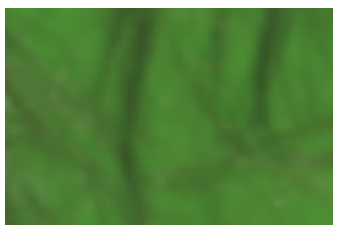

(d) Bilinear
Figure 3: A region with high frequency detail from original DPX video frame from the 'face' sequence (a) and the corresponding region generated by expansion of the halfresolution video frame by our algorithm (b), as well as by B-Spline interpolation (c) and Bilinear interpolation (d).

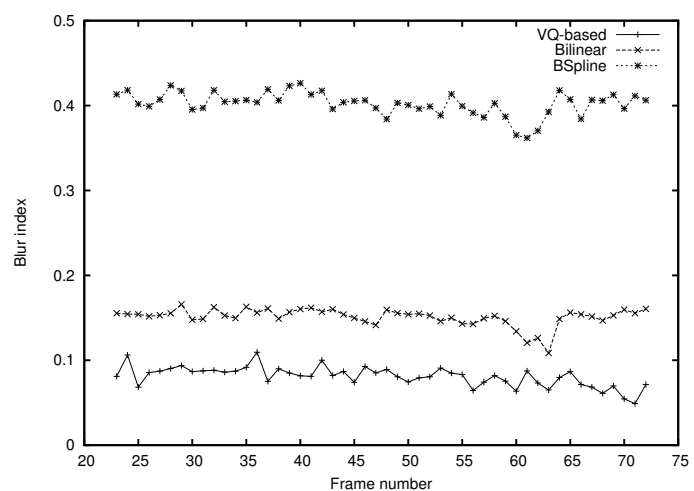

Figure 4: Frame-by-frame blurriness comparison between sequence of images (from 'outdoor' sequence) expanded with VQ-based, bilinear, and b-spline methods.

forms conventional approaches based on both objective and subjective metrics, with PSNR and WPSNR scores almost double that of the best conventional approaches. We hope to continue working on our algorithm which is still in its preliminary stages by

1. Learning statistical co-occurrence of neighbouring codebook blocks in images

2. Mediating the addition of high frequency predicted detail with the energy of the underlying region in the image to prevent prediction of detail in the absence of high frequency information in the original image.

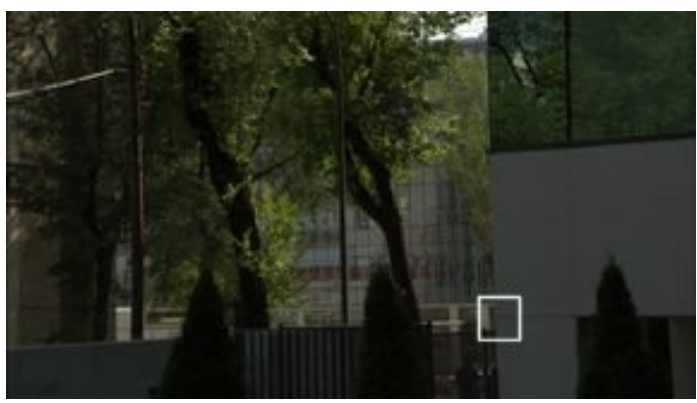

Figure 5: Image taken outside in bright light, with sharp focus containing more high frequency detail. Box shows area used in Figure 6.

\section{ACKNOWLEDGEMENTS}

This work is supported by the European Commission under the IP-RACINE project (IST-2-511316-IP).

\section{REFERENCES}

Barnsley, M. and Hurd, L. (1993). Fractal image compression. AK Peters.

Chaddha, N., Vishwanath, M., and Chou, P. (1995). Hierarchical vector quantization of perceptually weighted block transforms. Proceedings of the Conference on Data Compression.

Girod, B. (1993). What's wrong with mean-squared error? In Watson, A. B., editor, Digital Images and Human Vision, pages 207-220.

Gunawan, I. P. and Ghanbari, M. (2005). Image quality assessment based on harmonics gain/loss information. In Proceedings of ICIP'05 (IEEE International Conference on Image Processing), volume 1, pages 429-432, Genoa, Italia.

Levine, J., Mason, T., and Brown, D. (1992). lex \& yacc. O'Reilly \& Associates, Inc. Sebastopol, CA, USA.

Linde, Y., Buzo, A., and Gray, R. (1980). An Algorithm for Vector Quantizer Design. IEEE Transactions on Communications, 28(1):84-95.

Pratt, W. K. (1978). Digital Image Processing. John Wiley and Sons.

Voloshynovskiy, S., Herrigel, A., Baumgärtner, N., and Pun, T. (1999). A stochastic approach to content adaptive digital image watermarking. In International Workshop on Information Hiding, volume LNCS 1768 of Lecture Notes in Computer Science, pages 212-236, Dresden, Germany. Springer Verlag. 


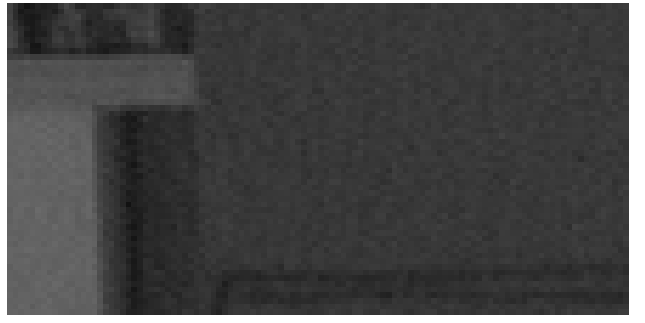

(a) Original

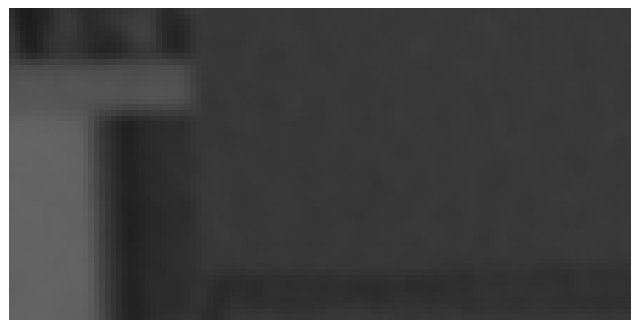

(c) Bspline

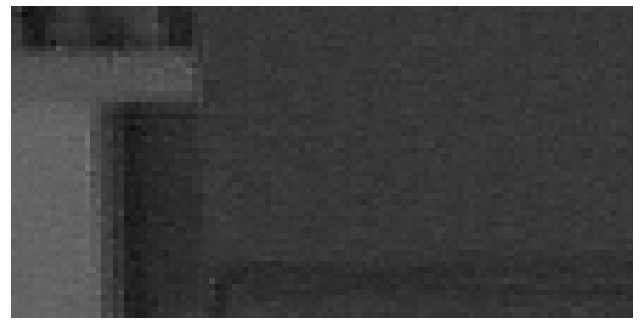

(b) Our algorithm

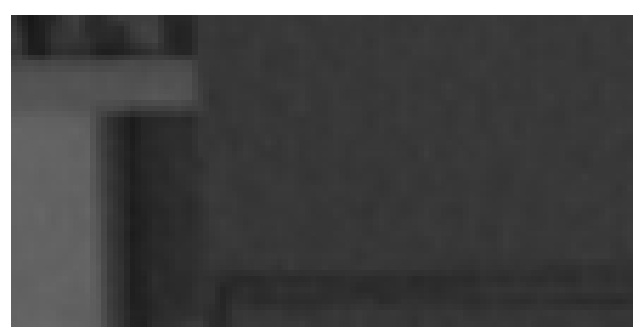

(d) Bilinear

Figure 6: Samples taken from the frame shown in Figure 5. The algorithm convincingly synthesises speckle on the concrete wall but leaves the white wall in the background speckle free.

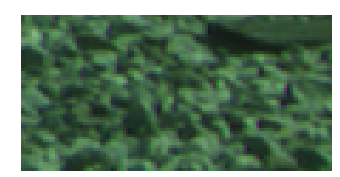

(a) Original

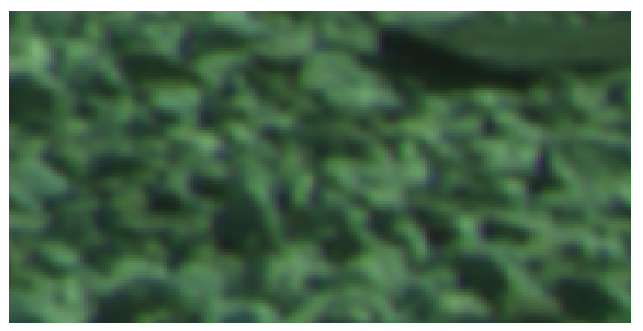

(c) Bspline

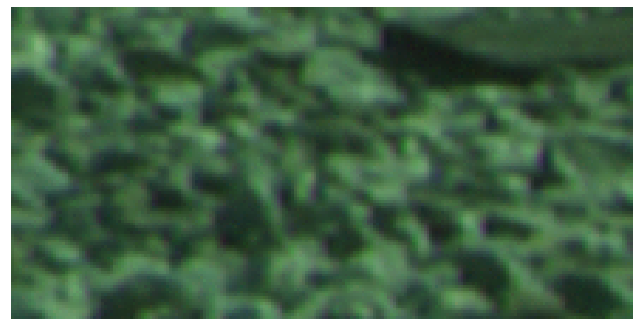

(b) Our algorithm

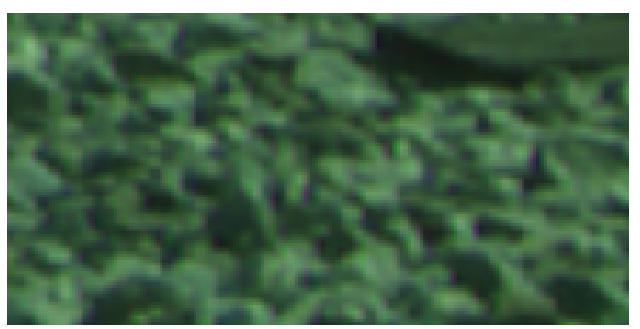

(d) Bilinear

Figure 7: Samples from 'train' sequence. The original patch (a) is taken from $2 \mathrm{~K}$ resolution image. The patches from the expanded images (b,c, and d) are of $4 \mathrm{~K}$ resolution. 